\title{
Dosificación mínima del reactivo Fenton para la remediación de agua contaminada con colorantes
}

\section{(Fenton's reagent minimum dosage for remediation of water contaminated with dyes)}

\author{
Gina Terán ${ }^{1}$, Paola Posligua ${ }^{1}$, Carlos Banchón ${ }^{1}$
}

\begin{abstract}
Resumen:
Efluentes de la industria textil no solo representan un riesgo latente para la biodiversidad en nuestro planeta sino también para el ser humano debido a la contaminación generada por colorantes industriales. En el presente estudio se evaluó un proceso Fenton para la decoloración de agua contaminada con amarillo 160, azul 81 y rojo 190 con una concentración inicial de $3300 \mathrm{mg} \cdot \mathrm{L}^{-1}$ y una demanda química de oxígeno de $1719 \mathrm{mg} \mathrm{O}_{2} \cdot \mathrm{L}^{-1}$. Cambios de $\mathrm{pH}$ y dosis molares de sulfato ferroso y peróxido de hidrógeno fueron evaluados. El proceso Fenton permitió un 99,9\% de remoción de materia orgánica y $100 \%$ de remoción de turbidez cuando se trabajó con $\mathrm{pH}$ de 3,5 y dosis molar $\mathrm{Fe}^{+2} / \mathrm{H}_{2} \mathrm{O}_{2}$ entre $1: 3$ y 1:5. Mediante un barrido espectrofotométrico y medición del potencial de oxido-reducción, se demostró que la calidad del agua descontaminada se asemejó a la del agua ultrapura tipo I.
\end{abstract}

Palabras clave: colorantes azoicos; potencial de oxido-reducción; Pelileo; industria textil

\begin{abstract}
:
Effluents from the textile industry not only represent a latent threat to biodiversity on our planet but also to humans due to the pollution generated by industrial dyes. In this study a Fenton process was evaluated for the decoloration of water contaminated with yellow 160, blue 81 and red 190 with an initial concentration of $3300 \mathrm{mg}^{-\mathrm{L}^{-1}}$ and a chemical oxygen demand of 1719 $\mathrm{mg} \cdot \mathrm{L}^{-1}$. Changes in $\mathrm{pH}$ and molar doses of ferrous sulfate and hydrogen peroxide were evaluated. The Fenton process allowed $99,9 \%$ removal of organic matter and $100 \%$ removal of turbidity when it worked at $\mathrm{pH} 3,5$ and molar dose $\mathrm{Fe}^{+2} / \mathrm{H}_{2} \mathrm{O}_{2}$ between 1:3 and 1:5. By spectrophotometric scanning and measurement of redox potential, it was shown that the quality of decontaminated water resembled the ultrapure water type I.
\end{abstract}

Keywords: azoic dyes; oxidation-reduction potential; Pelileo; textile industry

\section{Introducción}

El Programa de las Naciones Unidas para el Medio Ambiente estableció que la industria a nivel mundial es responsable de descargar entre 300 a 500 millones de toneladas anuales de efluentes con metales pesados, lodos y colorantes en cuerpos de agua (Roy Choudhury, 2013). En la industria textil, se han reportado consumos anuales de alrededor de $7 \times 10^{5}$ ton de colorantes y consumos de agua de hasta $150 \mathrm{~L}$ por cada $\mathrm{kg}$ de producto textil (Körbahti y Tanyolaç, 2008; Eslami, A., Moradi, M., Ghanbari, F., \& Mehdipour, F., 2013; Garcés y Hernández, 2012; Bouasla, 2012; Bahmami, Rezaei, Esrafili, Gholami y Jonidi, 2013). Se estima que de la cantidad utilizada

\footnotetext{
${ }^{1}$ Universidad de Las Américas, Quito - Ecuador ( \{gteran, paola.posligua, c.banchon\} @udlanet.ec )
} 
de colorantes en la industria textil, aproximadamente se desperdicia hasta un $15 \%$ de colorantes en efluentes (Daud, Akpan, y Hameed, 2012; Cui, Li, Zhao y Han, 2014); en Malasia el 22\% del volumen total de aguas residuales corresponde a la industria textil (Daud, Akpan, y Hameed, 2012). Con respecto al impacto ambiental, del total de colorantes azoicos, 3000 están catalogados como cancerígenos y mutagénicos, debido a su descomposición en aminas aromáticas (GrekovaVasileva y Topalova, 2009; Lau et al., 2014). A nivel local, un escenario de contaminación es el efluente descargado por la industria textil en los afluentes del río Patate en el cantón Pelileo (Ambato-Ecuador), cauce donde se reportó una demanda bioquímica de oxígeno de 450 mg. $\mathrm{L}^{-1}$, una demanda química de oxígeno de $880 \mathrm{mg} \cdot \mathrm{L}^{-1}$ y con coloración azul intensa (El Telégrafo, 2009). Se estima que aproximadamente 44 lavanderías de jeans en Pelileo consumen aproximadamente hasta 30 L.s ${ }^{-1}$ de agua para la producción diaria de textiles (EI Telégrafo, 2013). A la fecha, en Ecuador, no se han reportado acciones de remediación a gran escala que aporten con la solución de esta problemática.

Entre las tecnologías de remediación de efluentes industriales, las más comunes son coagulaciónfloculación, coagulación con extractos de plantas, electrocoagulación, adsorción y tratamientos biológicos (Barbusiński, 2005; Moreno, Figueroa y Hormaza, 2013; Gaber, Abu Ghalwa, Khedr, y Salem, 2013; Cui, Li, Zhao y Han, 2014; Revelo, Proaño, y Banchón, 2015). El proceso Fenton se destaca porque éste oxida completamente residuos colorantes; otras tecnologías como las biológicas, generan oxidaciones incompletas de los colorantes, es decir producen compuestos orgánicos intermediarios con potencial tóxico para el medio ambiente. Desde su descubrimiento en 1890 por Henry John Horstman Fenton, el reactivo Fenton -peróxido de hidrógeno y hierro como catalizador- es capaz de oxidar compuestos alifáticos y aromáticos halogenados, nitroaromáticos, colorantes azo, fenoles halogenados, formaldehído, herbicidas y hexadecano (Litter, Domènech, y Jardim, 2001). El proceso de Fenton también es aplicable para aguas residuales de la industria alimenticia; por ejemplo a escala piloto se utilizó la reacción de Fenton para aguas contaminadas con tartrazina con eficiencias de remoción de hasta el 99\% (Rojas, Giraldo y Trujillo, 2009). Se han reportado procesos de oxidación avanzada donde se potencia la reacción Fenton con energía ultravioleta. Por ejemplo, en el tratamiento de aguas residuales contaminadas con naranja 2, rojo ácido 151, naranja ácido 7, rojo 88 y azul ácido 113 se alcanzaron porcentajes de remediación próximos a 100 en tiempos de reacción de hasta 60 minutos (Riaza Frutos, Manzano, \& Quiroga, 2007; Ohura, Harada, Shiki, Kawakita y Biswas, 2012).

En Ecuador, se ha empleado el proceso Fenton para el tratamiento de suelos contaminados con hidrocarburos en Nueva Loja (Villacreces, 2013). Sin embargo, la aplicación de este proceso para el tratamiento de aguas residuales industriales no se ha difundido completamente a pesar de ser una técnica altamente efectiva y de bajo consumo de energía. A pesar del avance de la tecnología y de la modernización de la sociedad, el impacto ambiental y la contaminación generada por la 
actividad textilera es persistente. A causa de esto, se planteó en el presente trabajo el objetivo de evaluar las condiciones operacionales de un proceso Fenton para la oxidación de los colorantes reactivos amarillo 160, azul 81 y rojo 190, de aplicación industrial. En la presente investigación se experimentaron las condiciones mínimas y óptimas para la futura aplicación del proceso Fenton en el tratamiento de aguas residuales provenientes de actividades textileras artesanales.

\section{Metodología}

\subsection{Parámetros de caracterización físico-química del agua}

Las muestras de agua contaminada que se utilizaron en el presente estudio se prepararon con los colorantes azoicos reactivos industriales amarillo 160, azul 81 y rojo 190 provistos por Aromcolor S.A. (Quito, Ecuador). Estos colorantes se agregaron a $500 \mathrm{~mL}$ de agua destilada hasta obtener una concentración final de $3300 \mathrm{mg} \cdot \mathrm{L}^{-1}$; es decir, la concentración de cada colorante azoico en el agua fue de $1100 \mathrm{mg} \cdot \mathrm{L}^{-1}$.

La turbidez (NTU) fue medida mediante nefelometría (HANNA instrument, HI 88713, USA). El pH y el potencial de oxido-reducción (ORP) fueron medidos en un potenciómetro con electrodos de vidrio-platino (HANNA Instruments, HI 2550, HI 3131, USA). La demanda química de oxígeno (DQO) en $\mathrm{mg} \mathrm{O}_{2} \mathrm{~L}^{-1}$ fue determinada mediante espectrofotometría UV-VIS a $620 \mathrm{~nm}$ mediante el decrecimiento de la concentración de cromato luego de la oxidación de la materia orgánica con dicromato (VII) de potasio, ácido sulfúrico, y sulfato de plata-mercurio (Nanocolor, MachereyNagel COD Ref. 985 023, Alemania). La absorbancia en un rango de 190 a 1100 nm y el color fueron medidos en un espectrofotómetro UV-Vis (Nanocolor II, Macherey-Nagel, Alemania). La medida del color en el espectrofotómetro fue dada en unidades CIE L*a*b* (según la Comisión Internacional de la lluminación); estas unidades fueron transformadas a formato RGB (red, green, blue) mediante el software de libre acceso EasyRGB (colour calculator, http://www.easyrgb.com/).

\section{2. Índice del potencial de óxido-reducción}

Se evaluó la calidad de remoción de colorantes del agua tratada a través de la relación entre el ORP del agua tratada y el ORP del agua pura tipo I. A esta relación se la denominó índice del potencial de oxido-reducción.

\subsection{Proceso Fenton}

El proceso Fenton se llevó a cabo en un equipo para prueba de jarras (Velp scientific, JLTG, España) a una velocidad de 300 rpm y tiempo de mezcla de 45 minutos. Se utilizaron volúmenes de agua residual sintética de $250 \mathrm{~mL}$ y la temperatura inicial de todas las muestras fue de $40^{\circ} \mathrm{C}$. Todas las muestras se expusieron a una luminosidad de 30397.33 lux entre 3 a 24 horas. La luminosidad fue medida en un luxómetro (Sper scientific, 850007, U.S.A). Las muestras se filtraron en una bomba de vacío (Gast Manufacturing, DOA-P704 shown, U.S.A, 2002) y papel filtrante de 
0,5 $\mu \mathrm{m}$ (Microclar, Argentina). Finalmente, las muestras fueron caracterizadas según los parámetros físico-químicos antes descritos. El pH inicial fue modificado según los respectivos diseños experimentales con ácido sulfúrico e hidróxido de sodio.

\subsection{Dosificación del reactivo Fenton}

Para el cálculo estequiométrico del volumen de $\mathrm{H}_{2} \mathrm{O}_{2}$ a adicionar al medio de reacción fue necesario determinar el DQO inicial del agua contaminada (Eslami, A., Moradi, M., Ghanbari, F., y Mehdipour, F., 2013). Según la caracterización del agua residual, el DQO inicial tuvo un valor de $1719 \mathrm{mg} . \mathrm{L}^{-1}$. A partir de este dato de DQO, según Rodríguez (2010), se obtuvo que por cada litro de agua residual se adicionó $12,174 \mathrm{~mL}$ de peróxido de hidrógeno al 30\%. Las unidades experimentales en el presente proyecto fueron de $250 \mathrm{~mL}$; por tanto, se adicionaron 3,04 $\mathrm{mL}$ de peróxido de hidrógeno al $30 \%$ por cada unidad experimental. La dosificación de sulfato ferroso heptahidratado fue calculada en un rango molar $\mathrm{Fe}^{+2} / \mathrm{H}_{2} \mathrm{O}_{2}$ de 1:1 a 1:80. En la Figura 1 se observan las dosis másicas de sulfato de hierro (II) a adicionarse para cada relación molar.

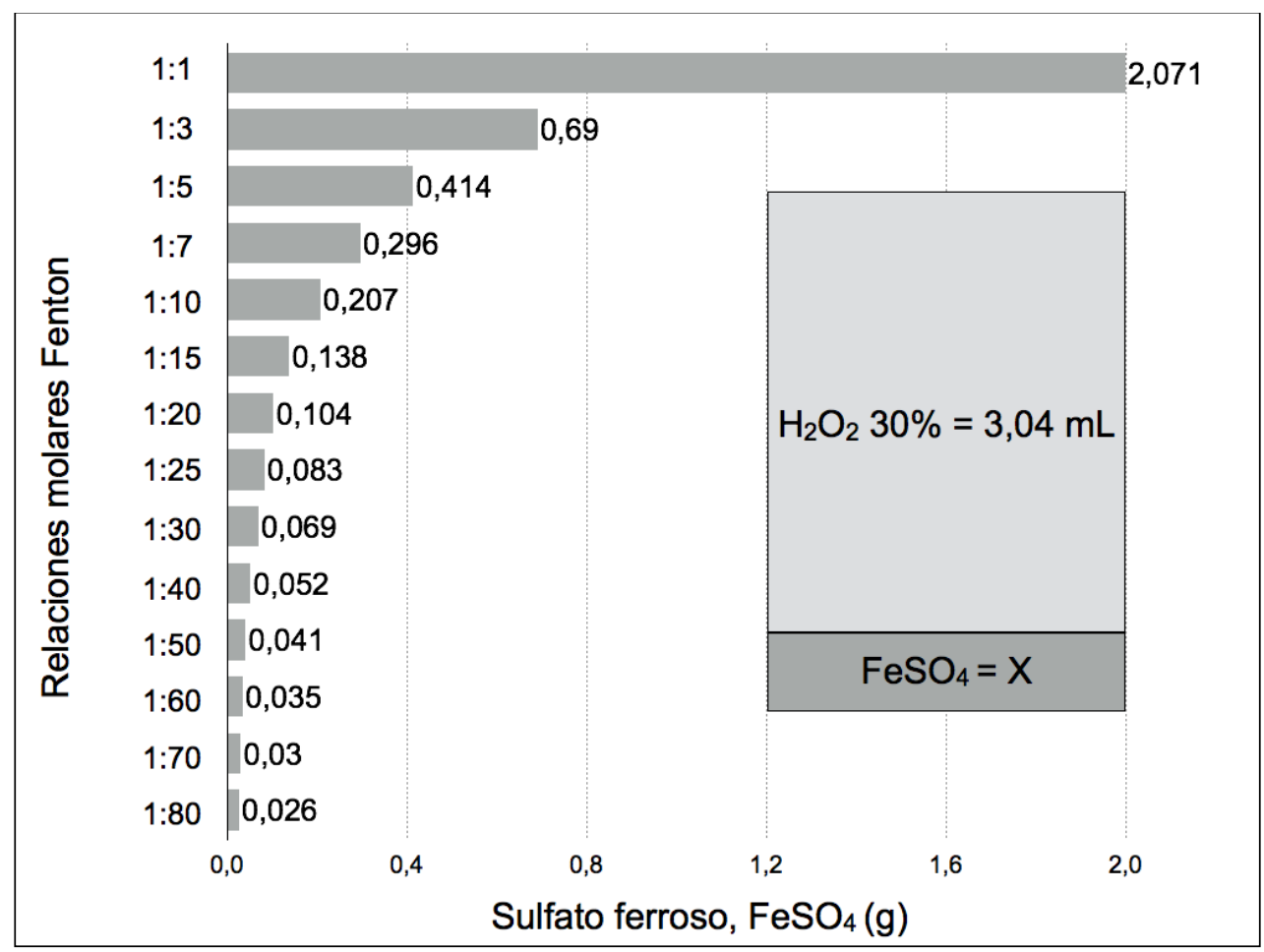

Figura 1. Relaciones molares Fenton entre 1:1 hasta 1:80 según diseño experimental exploratorio y cantidades de sulfato ferroso a adicionar por cada 3,04 mL de peróxido de hidrógeno al $30 \%$ a $250 \mathrm{~mL}$ de agua residual sintética.

\subsection{Diseño experimental exploratorio}

En el presente estudio se desarrolló un diseño experimental exploratorio (DEE) para determinar la dosis molar óptima del reactivo Fenton en función del cambio de color y cambio de potencial de oxido-reducción (ORP). El DEE se utilizó para la evaluación de la influencia de las variables operacionales (dosis de reactivo Fenton y $\mathrm{pH}$ ). En la Figura $2 A$, se presenta el DEE en donde $X_{1}$ representa la dosis de reactivo Fenton, $X_{2}$ el $\mathrm{pH}$ inicial y la respuesta representada por $y$ (cambio 
de ORP, cambio de color). En la Figura 2B, de los resultados del primer diseño experimental se seleccionaron los mejores rangos de dosificación para luego optimizar el proceso Fenton. En la optimización se combinaron las tres variables independientes. Donde, $X_{3}$ dosis de reactivo Fenton, $X_{4} \mathrm{pH}$ inicial, $X_{5}$ tiempo de reposo, versus la respuesta $y^{\prime}$ (cambio de ORP, cambio de color).

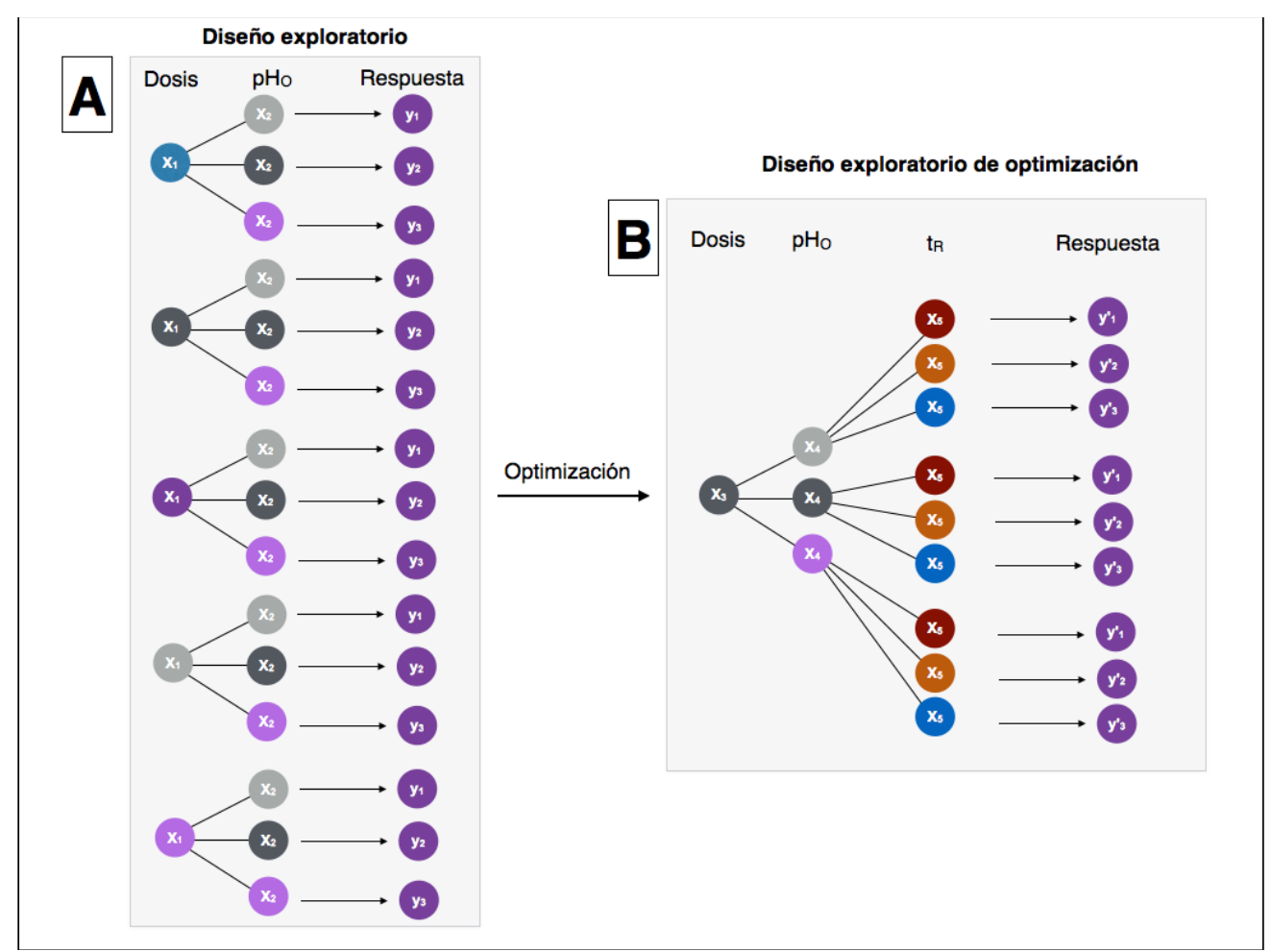

Figura 2. Diseño experimental exploratorio $(A)$ para la búsqueda de dosis del reactivo Fenton para la decoloración del agua contaminada y Diseño experimental exploratorio (B) para la optimización de la dosificación del reactivo Fenton.

\section{Resultados}

Todos los resultados obtenidos de los respectivos tratamientos se basaron en cálculos estequiométricos tomando en cuenta un $\mathrm{DQO}$ del agua residual de $1719 \mathrm{mg} \mathrm{O}_{2} \mathrm{~L}^{-1}$. El estudio del proceso de remediación tuvo como primer objetivo evaluar las condiciones de pH inicial del agua contaminada, así como la dosificación a diferentes rangos del reactivo Fenton en función del ORP (Figura 3). Con propósitos de comparación, en la Figura 3 se presentan los valores de ORP del agua tipo I (124,4 mV) y del agua residual sintética $(224,4 \mathrm{mV})$.

Según el diseño experimental exploratorio desarrollado para el presente trabajo, se propone una sucesión en cadena de experimentos que tiene como propósito la evaluación de las condiciones óptimas de remediación. Es así que, según los resultados de la Figura 3, se prosiguió a experimentar con dosis molares entre $1: 3$ a 1:10, puesto que la dosis molar 1:5 fue la que permitió que el agua tratada se asemeje en su potencial de óxido-reducción al agua pura tipo I. Para corroborar la eficacia de las dosis molares, además se midió la absorbancia y el color del agua tratada mediante espectrofotometría. Por lo tanto, se evaluó la reacción Fenton a diferentes pH a dosis óptimas entre 1:3 a 1:10 (Figura 4). 


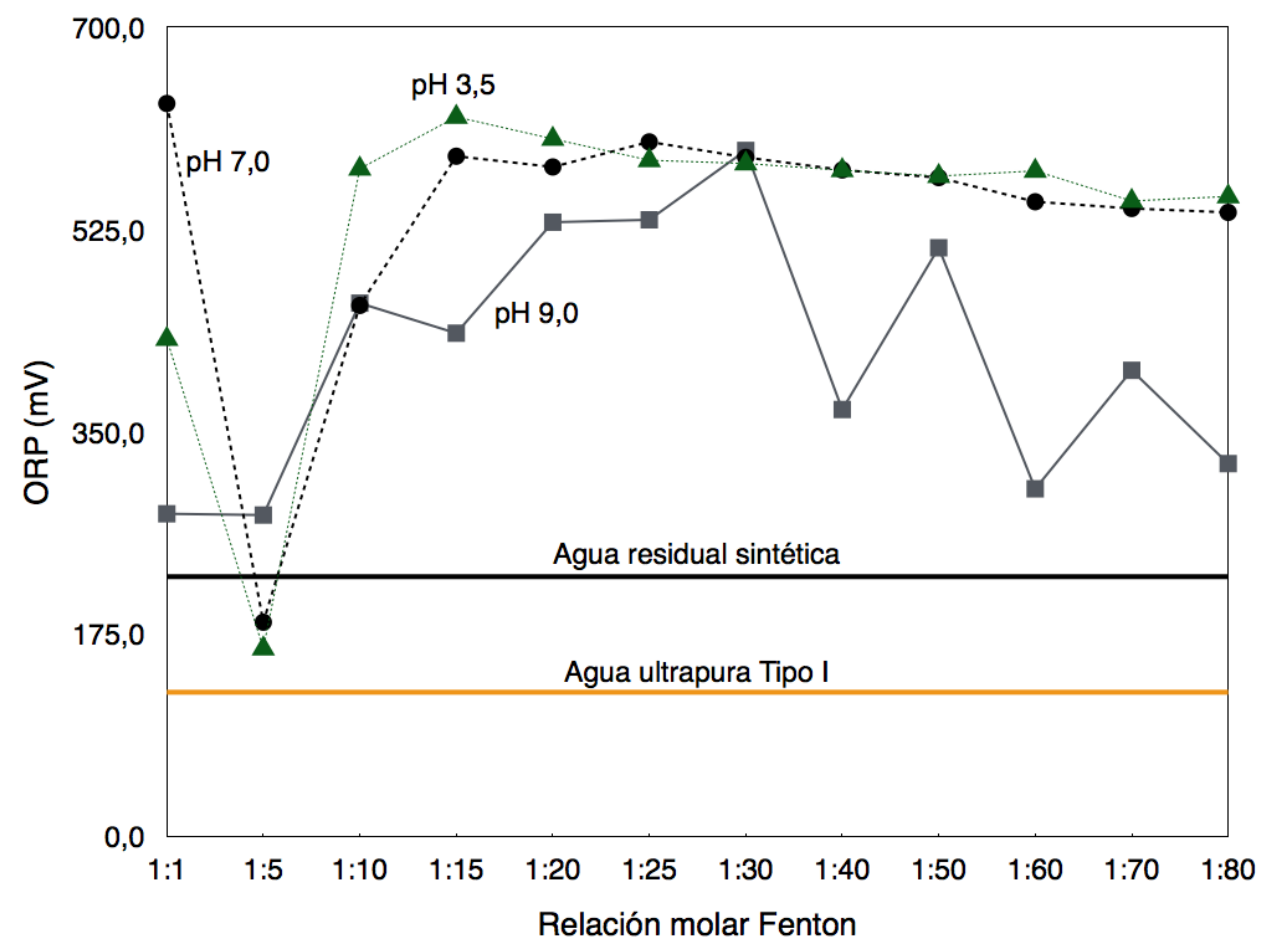

Figura 3. Mediciones del potencial de oxido-reducción (ORP) para agua residual sintética, agua ultrapura tipo I y muestras de agua tratada con dosis molares de Fenton entre 1:1 hasta 1:80.

En donde, $(\boldsymbol{\Lambda})$ muestras tratadas a pH inicial ácido, $(\bullet)$ muestras tratadas a pH inicial neutro y $(\square \square)$ muestras tratadas a pH inicial básico.

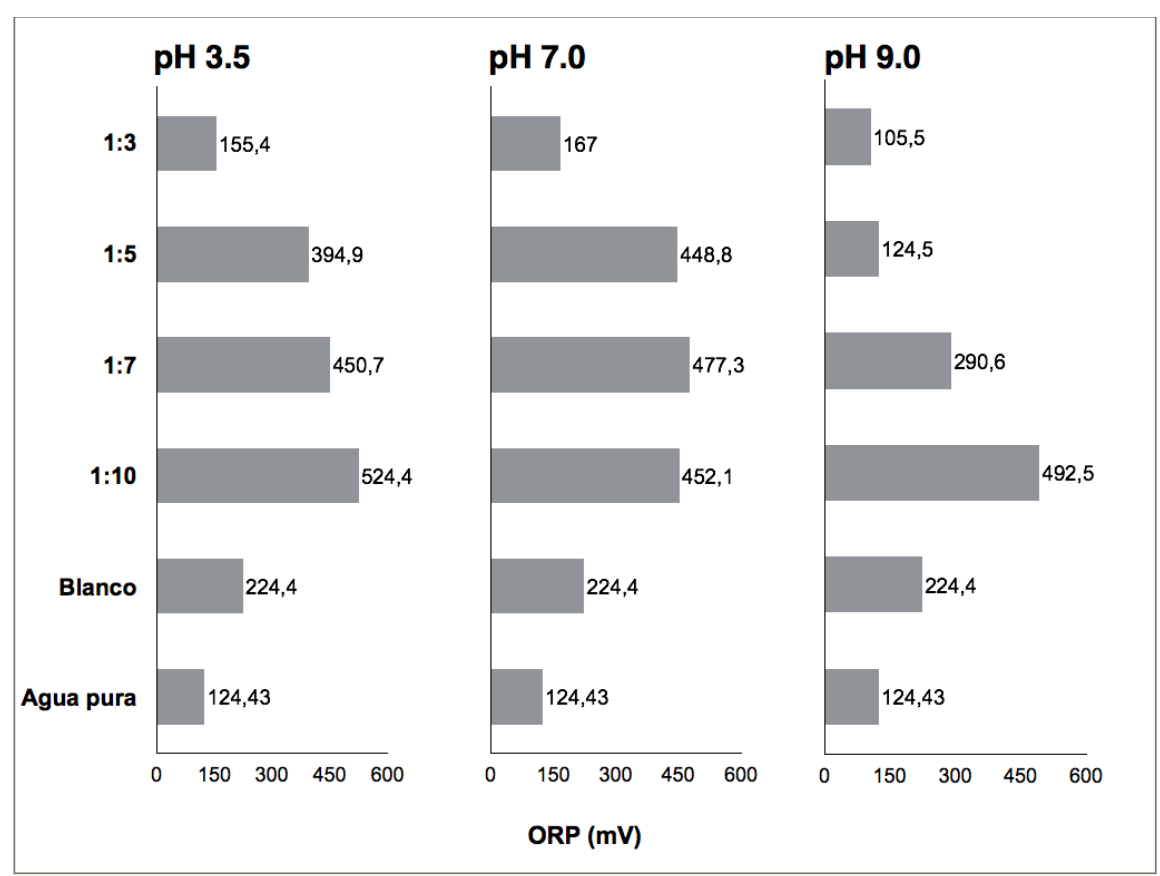

Figura 4. Mediciones del potencial de oxido-reducción (ORP) para agua residual sintética (Blanco), agua ultrapura tipo I (Agua pura) y muestras de agua tratada con dosis molares de Fenton entre 1:1 hasta 1:10 a $\mathrm{pH}$ inicial ácido, básico y neutro.

De acuerdo con los resultados obtenidos en la Figura 4 sobre el cambio de ORP y el análisis colorimétrico de las muestras de agua tratada (Tabla 1), se comprobó que la mejor dosis fue la relación molar $\mathrm{Fe}^{+2} / \mathrm{H}_{2} \mathrm{O}_{2} 1: 3$ a un $\mathrm{pH}$ inicial de 3,5. Un análisis espectrofotométrico también 
comprueba la decoloración del agua residual sintética bajo estas condiciones al compararse los barridos de absorbancia de muestras de agua pura tipo I, agua contaminada y tratada mediante Fenton (Figura 5). Según los resultados de las figuras 3 y 4 , el pH inicial del agua contaminada que dio óptimos resultados en función del índice ORP fue 3,5 en concordancia con otros autores (Benatti, C. T., \& Tavares, C. R. G., 2012; Bouasla, C., Ismail, F., \& Samar, M. E.-H., 2012; Daud, N. K., Akpan, U. G., \& Hameed, B. H., 2012; Patel \& Patel, 2013).

Tabla 1. Mediciones de color en unidades CIE $L^{*} a^{*} b$ para los tratamientos a dosis molares entre 1:1 hasta $1: 80 \mathrm{a} \mathrm{pH} 3,5$ y 24 horas como tiempo de reposo, color del agua residual y color del agua pura tipo I.

\begin{tabular}{|c|c|c|c|}
\hline Muestra & $\mathbf{L}$ & $\mathbf{a}$ & $\mathbf{b}$ \\
\hline Agua residual & 0,4 & 1,6 & 0,4 \\
\hline Agua pura tipo I & 100 & 0 & 0 \\
\hline Tratamiento $1: 1$ & 92,9 & 5,9 & 19,2 \\
\hline Tratamiento 1:3 & 99,4 & 0 & 2,1 \\
\hline Tratamiento 1:5 & 97,7 & 0,7 & 1,1 \\
\hline Tratamiento 1:7 & 98,3 & 0,5 & 1,8 \\
\hline Tratamiento $1: 10$ & 95,2 & 0,1 & 0,7 \\
\hline Tratamiento $1: 15$ & 99,8 & 0 & 1,4 \\
\hline Tratamiento $1: 20$ & 99,5 & 0 & 3,1 \\
\hline Tratamiento $1: 25$ & 99,6 & 0,1 & 1,3 \\
\hline Tratamiento $1: 30$ & 99,9 & 0,1 & 1,8 \\
\hline Tratamiento $1: 40$ & 99,6 & 0,1 & 0,6 \\
\hline Tratamiento $1: 50$ & 95 & 0 & 0,9 \\
\hline Tratamiento $1: 60$ & 98,7 & 0,5 & 2,4 \\
\hline Tratamiento 1:70 & 97,6 & 1,1 & 4,6 \\
\hline Tratamiento 1:80 & 98,7 & 0,7 & 2,3 \\
\hline
\end{tabular}

Se experimentó la reacción Fenton a pH inicial 3,5 y relación molar 1:3, además de tratamientos químicos de oxidación con hipoclorito sódico $5 \%$ y peróxido de hidrógeno al $30 \%$ con propósitos comparativos. En la Figura 6 se observan los resultados de las mediciones del DQO, turbidez y ORP antes y después del respectivo tratamiento. En la Figura 5 también se comparan estos tratamientos con la medición de la absorbancia en rangos de longitud de onda entre 190-1100 nm.

\section{Discusión}

Según la Figura 8, la dosis molar óptima de $\mathrm{Fe}^{+2} / \mathrm{H}_{2} \mathrm{O}_{2}$ fue 1:5 (414 mg $\mathrm{FeSO}_{4}$ por cada 3,04 mL de $\mathrm{H}_{2} \mathrm{O}_{2} 30 \%$ ), pues ésta decoloró al agua contaminada ( $\mathrm{pH}$ inicial 3,5 ) hasta llegar a un ORP similar al del agua pura; mientras que, aunque sí hubo una decoloración a dosis molares mayores que 1:10, la dosis 1:5 fue la mínima que permitió obtener un potencial de oxido-reducción similar al del agua tipo I. El ion ferroso al tener su rol de catalizador y reactivo limitante, su concentración debe ser la óptima en relación al peróxido de hidrógeno para la producción de los radicales hidroxilos (Bouasla, C., Ismail, F., y Samar, M. E.-H. 2012). A partir de los resultados experimentales obtenidos en la Figura 8, se experimentó con dosis entre 1:3 hasta 1:10 para 
comprobar si existe una dosis molar óptima todavía menor que 1:5 (Figura 9), tomando en cuenta que el pH controla la calidad de la reacción de oxidación.

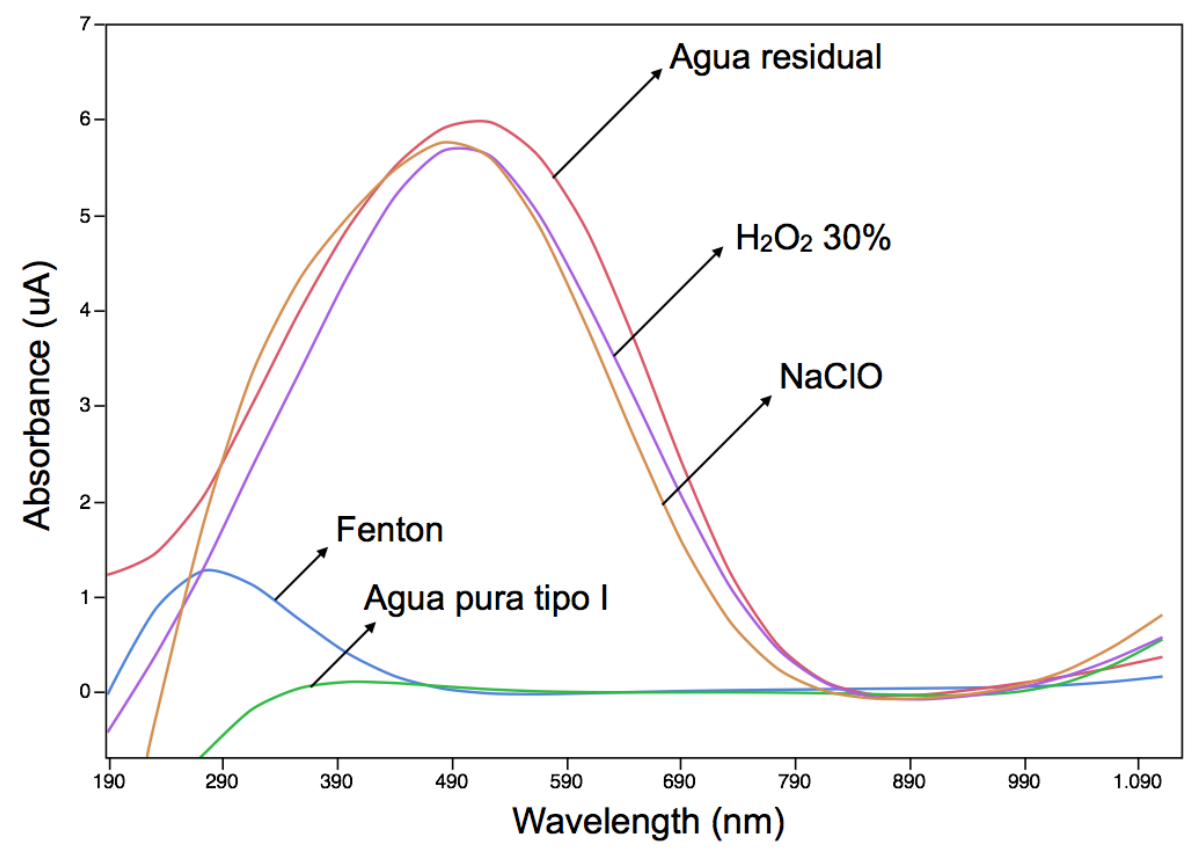

Figura 5. Absorbancia de muestras de agua pura tipo I, agua residual sintética, agua residual tratada con Cloro (hipoclorito de sodio 5\%), con Peróxido (peróxido de hidrógeno al 30\%) y Fenton a dosis molar 1:3 $\mathrm{pH} 3,5$ y 24 horas de reposo, rango de longitud de onda 190-1100 nm.
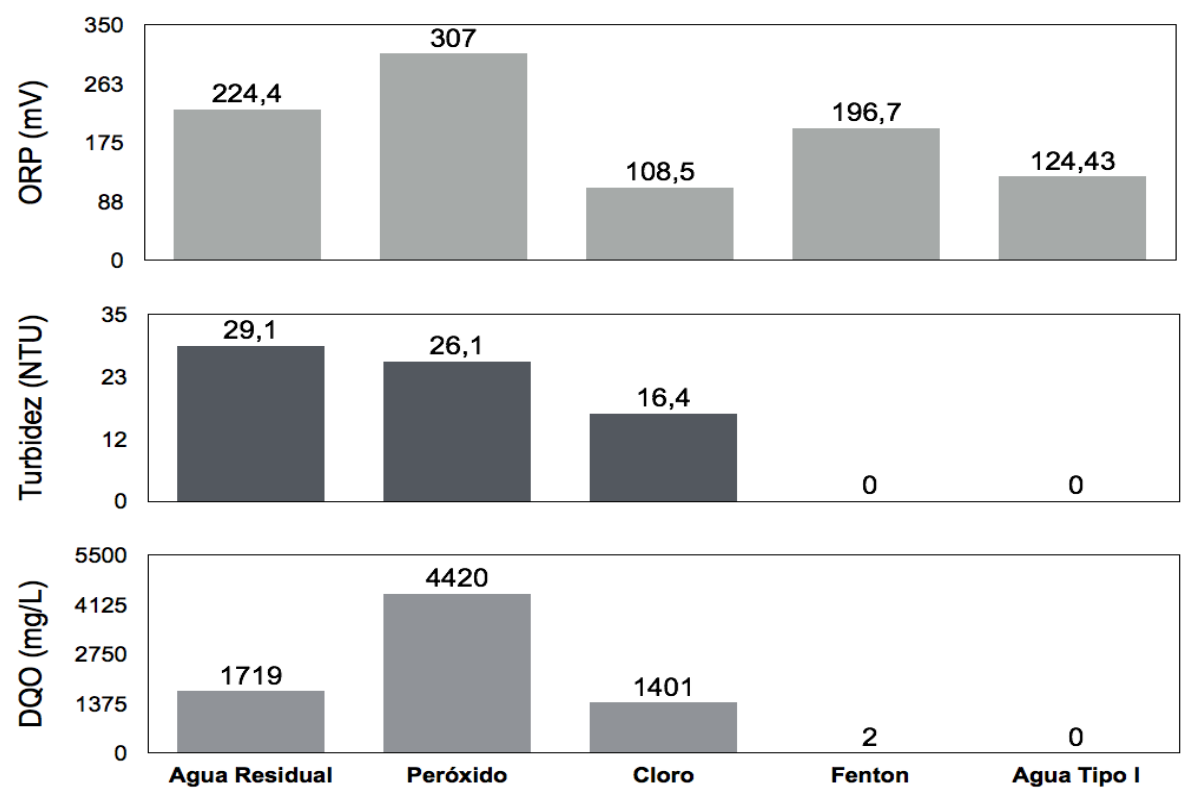

Figura 6. Caracterización físico-química (ORP, turbidez y DQO) del agua tratada mediante tres métodos: Fenton a dosis molar 1:3 pH 3,5 y 24 horas de reposo, Cloro (hipoclorito de sodio 5\%), Peróxido (peróxido de hidrógeno al 30\%).

Según la reacción (1), la acción catalizadora del ion ferroso produce la descomposición del peróxido de hidrógeno hasta la formación de radicales hidroxilos $\left(\mathrm{OH}^{\bullet}\right)$, los cuales son responsables de la decoloración del agua contaminada:

$$
\mathrm{Fe}^{+2}+\mathrm{H}_{2} \mathrm{O}_{2} \rightarrow \mathrm{Fe}^{+3}+\mathrm{OH}^{-}+\mathrm{OH}^{\bullet}
$$


La producción de radicales hidroxilos se ve afectada por el pH del medio y la concentración de peróxido de hidrógeno: conforme el pH tiende a la acidez, a mayor concentración de peróxido de hidrógeno la producción de radicales hidroxilos aumenta. Sin embargo, según los resultados experimentales (Figura 8) y revisión bibliográfica, altos volúmenes de peróxido de hidrógeno no aseguran la alta generación de radicales hidroxilos si acaso no se cumple la relación estequiométrica óptima con el ion ferroso (Bouasla, C., Ismail, F., y Samar, M. E.-H., 2012; Domínguez, J. R., Muñoz, M. J., Palo, P., González, T., Peres, J. A., y Cuerda-Correa, E. M., 2014). Es de notar que excesos de radicales hidroxilos $\left(\mathrm{OH}^{\bullet}\right)$ tienden a seguir oxidando iones ferrosos que no reaccionaron, promoviendo la formación de complejos de hidróxidos férricos:

$$
\mathrm{OH}^{\bullet}+\mathrm{Fe}^{+2} \rightarrow \mathrm{OH}^{-}+\mathrm{Fe}^{+3}
$$

Cabe mencionar también que aunque se acidificó el medio con ácido sulfúrico, no obstante, la acidificación del medio se debe también a la disolución de iones ferrosos, pues estos generan el aumento de la concentración de iones oxonios $\left(\mathrm{H}_{3} \mathrm{O}^{+}\right)$; estos oxonios debido a su naturaleza ácida ( $\mathrm{pK}=-1,7)$ son altamente reactivos y promueven la oxidación de compuestos orgánicos. En la presente investigación también se aplicó energía ultravioleta la cual participa en la producción de radicales hidroxilos así como iones ferrosos según Hansson, Kaczala, Marques, \& Hogland (2012):

$$
\mathrm{Fe}^{+3}+\mathrm{H}_{2} \mathrm{O}+h v \rightarrow \mathrm{OH}^{\bullet}+\mathrm{Fe}^{+2}+\mathrm{H}^{+}
$$

Según resultados experimentales (Figura 3), el pH óptimo inicial del agua contaminada fue 3,5 pues éste favorece la reacción de oxidación Fenton. Además, se determinó que las dosificaciones molares $\mathrm{Fe}^{+2} / \mathrm{H}_{2} \mathrm{O}_{2}$ entre 1:3 y 1:5 son las óptimas para la decoloración del agua contaminada (figuras 8 y 9 ).

El pH inicial 3,5 del agua contaminada regula la reacción de oxidación, pues existe una generación favorable de $\mathrm{OH}^{\bullet}$, los cuales son altamente oxidantes con un potencial de oxidación de 2,8 $\mathrm{V}$ y así permiten un mínimo del 90\% de decoloración y remoción de DQO bajo condiciones ácidas (Kalra, S. S., Mohan, S., Sinha, A., y Singh, G., 2011; Bouasla, C., Ismail, F., \& Samar, M. E.-H., 2012; Daud, N. K., Akpan, U. G., y Hameed, B. H., 2012). Sin embargo, se ha demostrado que un $\mathrm{pH}$ del agua contaminada por debajo de 3,0 no favorece su decoloración debido a la inhibición de la acción catalizadora del ion ferroso (Daud, N. K., Akpan, U. G., y Hameed, B. H., 2012; El Haddad et al., 2014). A pH mayor que 4 ocurre descomposición del peróxido de hidrógeno y se favorece que iones férricos formen complejos hidróxidos (reacción 4) con tendencia a precipitar y aumentar la producción de lodos (Benatti, C. T., y Tavares, C. R. G., 2012; Saber, A., Hasheminejad, H., Taebi, A., y Ghaffari, G., 2014).

$$
\mathrm{Fe}^{+3}+3 \mathrm{H}_{2} \mathrm{O} \rightarrow \mathrm{Fe}(\mathrm{OH})_{3}+3 \mathrm{H}^{+}
$$




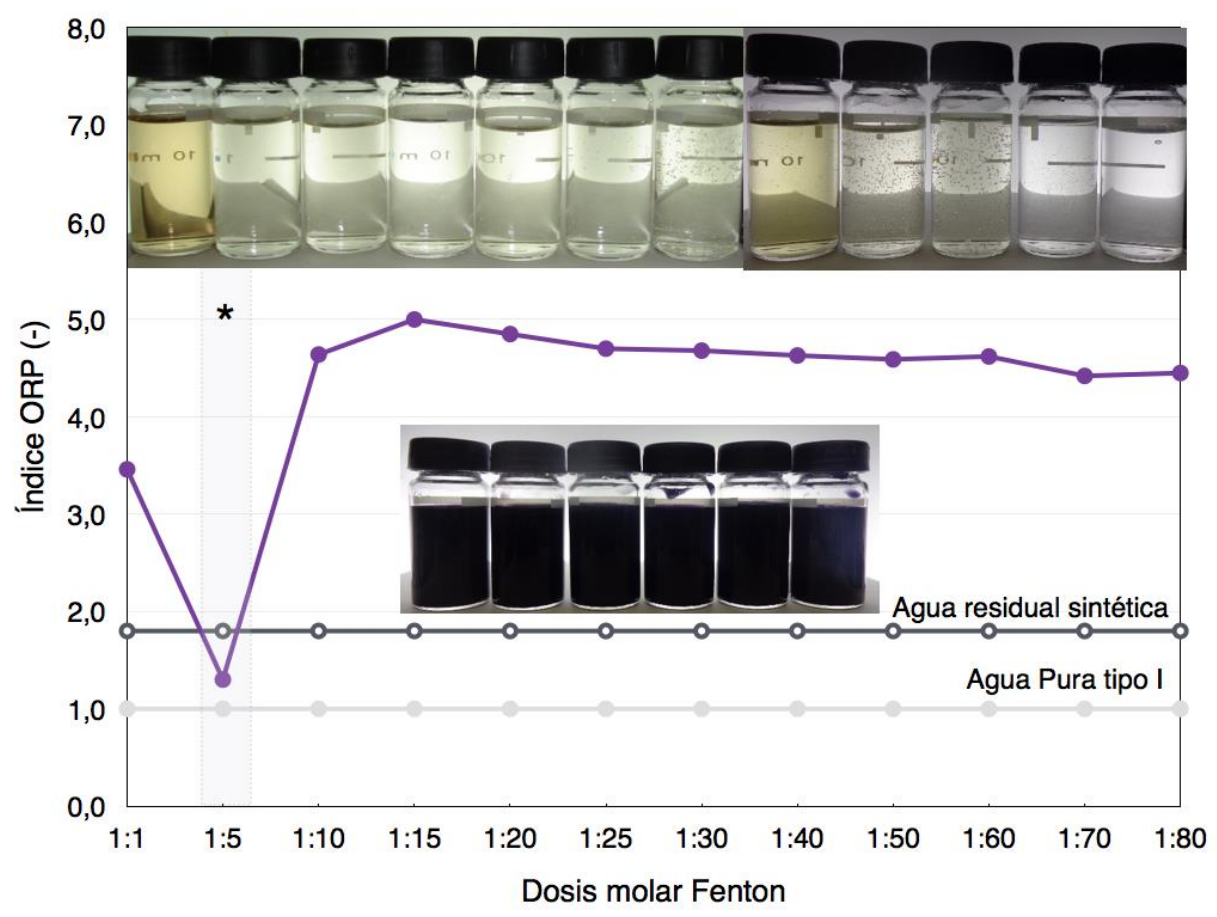

Figura 8. Índice ORP para el agua pura tipo I, agua residual sintética y tratamientos con dosis molares entre 1:1 hasta 1:80. En fotografías, las muestras tratadas con Fenton y la muestra original de agua residual sintética. Tratamientos Fenton a un $\mathrm{pH}$ inicial de 3,5 y tiempo de reposo de 24 horas.

En la Figura $9 B, C$ y $D$ se reportan los resultados de tratamientos con diversos oxidantes: reactivo Fenton (dosis 1:3, pH inicial 3,5), hipoclorito de sodio comercial al $5 \%$ y peróxido de hidrógeno al $30 \%$. De estos tratamientos, el reactivo Fenton permitió la remoción del $99,9 \%$ de DQO y 100\% de turbidez, en comparación con sus homólogos los cuales no removieron significativamente la contaminación del medio. Según los resultados de la Figura $9 B, C$ y $D$, los tratamientos con el reactivo Fenton evidencian el poder oxidativo de los radicales hidroxilos, en comparación con el potencial de oxidación del hipoclorito de sodio $5 \%$ (1,36 V) y peróxido de hidrógeno (1,78 V). Se comprueba que para este caso, el peróxido de hidrógeno al 30\% por si solo no es eficiente como oxidante de los colorantes contaminantes del agua (El Haddad, M., Regti, A., Laamari, M. R., Mamouni, R., y Saffaj, N., 2014).

La decoloración del medio contaminado fue comprobada mediante un barrido de absorbancia en un rango de longitud de onda entre 190 -1100 nm (Figura 5). Se demostró que en efecto la dosis molar 1:3 removió aquellos picos de absorbancia adjudicados a los colorantes amarillo 160, azul 81 y rojo 190. Además, mediante un análisis colorimétrico (Tabla 1) se determinó el color en unidades RGB (Figura 10) de los tratamientos con dosis Fenton entre 1:1 hasta 1:80. En la Figura 10 se observa el cambio de coloración de la muestra de agua pura tipo I, su contaminación con los tres colorantes y su decoloración mediante Fenton. Los resultados del análisis colorimétrico fueron sometidos a un análisis de varianza en el software R-project y como resultado se obtuvo que las dosis molares 1:5 y 1:10 tuvieron un p-estadístico mayor que 0,05. Es decir, el color final 
de estas muestras tratadas con dosis molares 1:5 y 1:10 no variaron significativamente del color de la muestra original de agua pura tipo I.

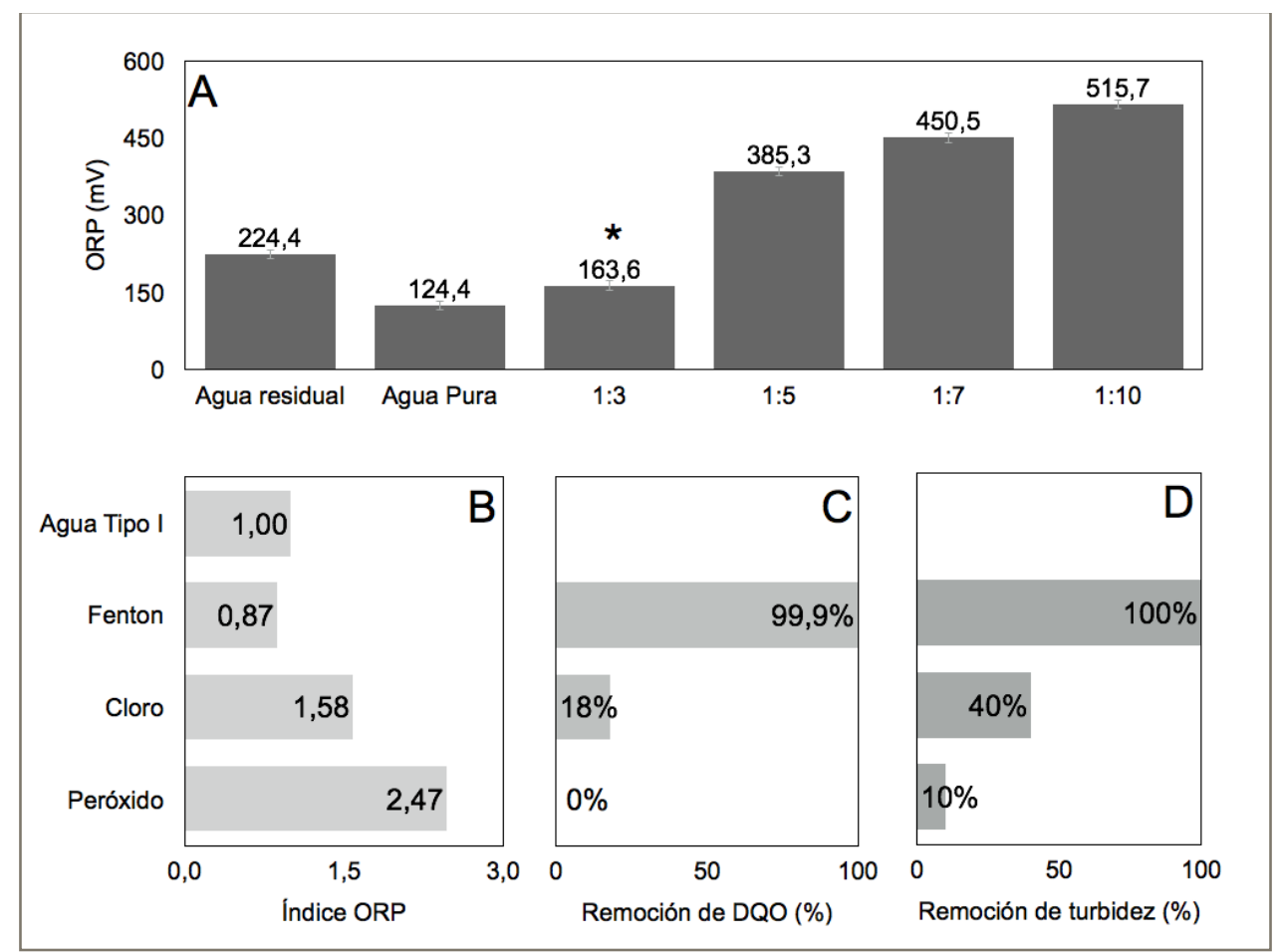

Figura 9. (A) Mediciones de ORP para los tratamientos Fenton de optimización en dosis molares entre 1:3 hasta 1:10, a pH inicial 3,5 y tiempo de reposo de 24 horas. (B) Índice ORP para agua pura tipo I, tratamiento Fenton con dosis molar 1:3 a pH inicial 3,5 y tiempo de reposo de 24 horas; (C) porcentajes de remoción de DQO para las muestras tratadas con Fenton, hipoclorito sódico $5 \%$ y peróxido de hidrógeno 30\%; (D) porcentaje de remoción de turbidez para las muestras tratadas con Fenton, hipoclorito sódico $5 \%$ y peróxido de hidrógeno $30 \%$.

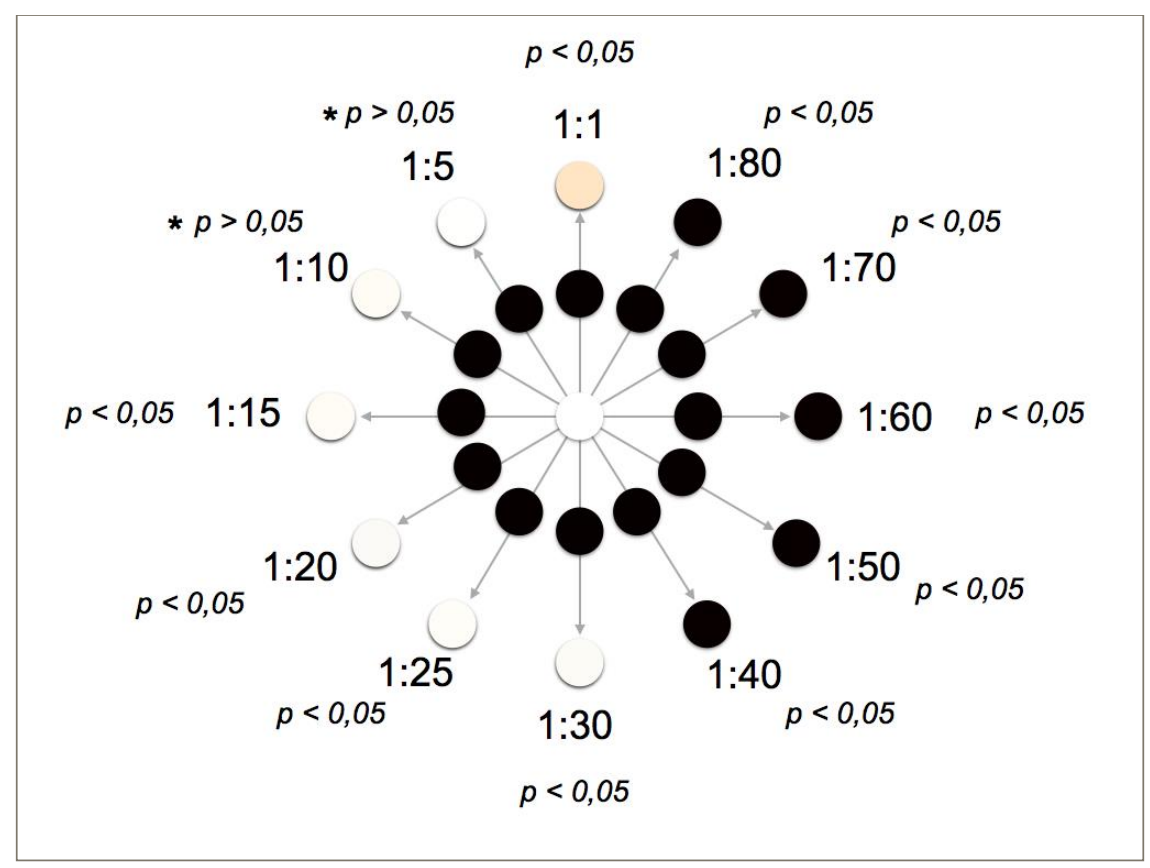

Figura 10. Representación gráfica del cambio de color tomando como referencia las transformaciones del color en unidades RGB (calculadas a partir de Tabla 1). En el centro se representa el color del agua pura tipo I, y a su alrededor los colores del agua residual sintética. En la periferia de la gráfica se representan los colores finales después del tratamientos. Se representa también el valor del p-estadístico de cada muestra tratada con Fenton y en referencia al valor del color del agua pura tipo I. 
Un análisis colorimétrico para los tratamientos entre 1:1 hasta 1:10 (Figura 11) determinó el color en unidades RGB así mismo determinó la varianza estadística entre el agua tratada y el agua pura tipo I. Como se puede observar en la Figura 11, el color final del agua tratada con dosis molares 1:3 y 1:5 no difiere estadísticamente del color del agua pura $(p>0,05)$. Este análisis colorimétrico, además del barrido de absorbancia, y las mediciones del índice ORP, comprueba que la dosis mínima y óptima para oxidar colorantes de agua contaminada con amarillo 160, azul 81 y rojo 190 (conc. final 3300 mg. $\mathrm{L}^{-1}$ ) fue una dosis molar $\mathrm{Fe}^{+2} / \mathrm{H}_{2} \mathrm{O}_{2}$ de 1:3.

En el presente y demás estudios se ha comprobado que la oxidación de colorantes mediante Fenton depende de la dosis molar $\mathrm{Fe}^{+2} / \mathrm{H}_{2} \mathrm{O}_{2}$ y el $\mathrm{pH}$ inicial; sin embargo, todavía se discute si el mecanismo de oxidación se refiere a la producción de radicales hidroxilos o a un mecanismo mediante la formación de especies del ion ferril [Fe(IV)O $]^{+2}$ (Chemizmu, K., y Fentona, R., 2009).

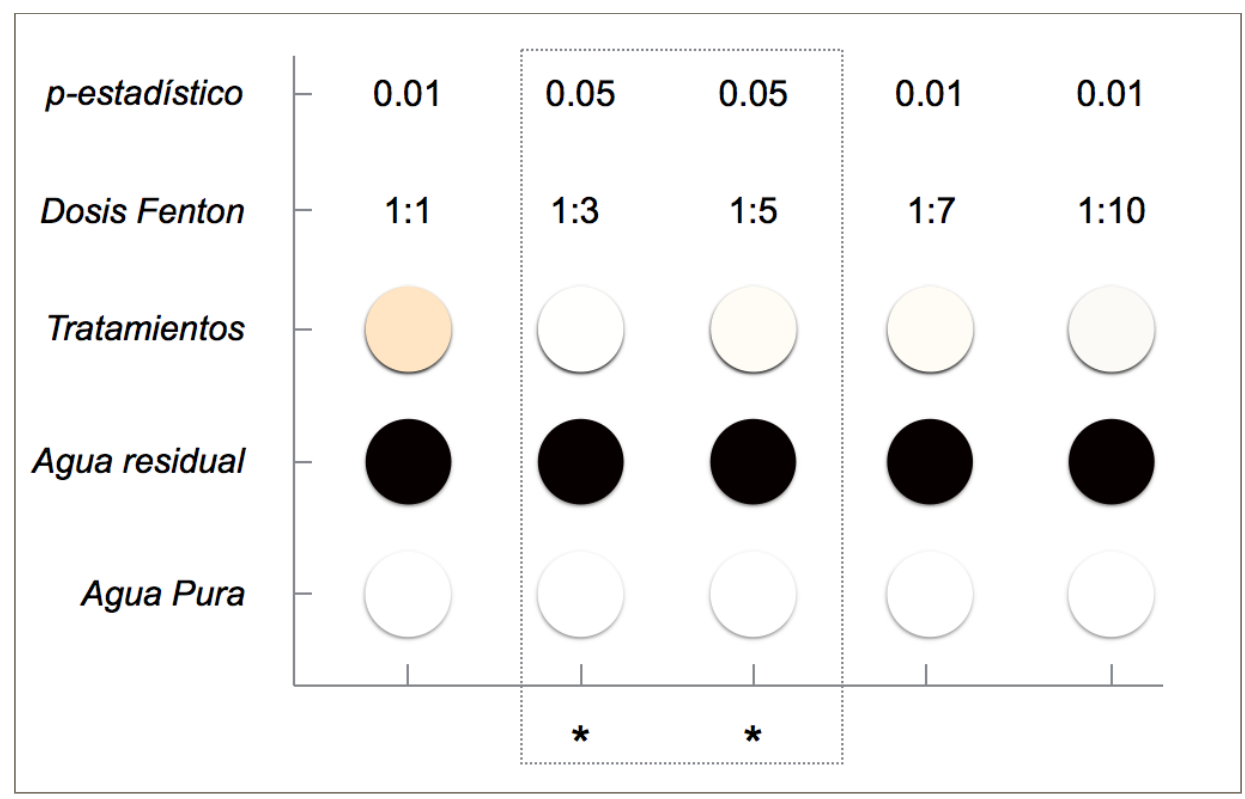

Figura 11. Representación gráfica de la optimización de dosis molares Fenton entre 1:3 hasta 1:10 versus los colores de cada muestra (colores en unidades RGB a partir de datos de Tabla 1), en comparación con los colores del agua residual sintética, y del agua pura tipo I. En la parte superior se presenta el valor del pestadístico tomando como referencia el valor del color de cada muestra y en referencia al valor del color del agua pura tipo I. Tratamiento a pH inicial 3,5 y 24 horas de reposo.

\section{Conclusiones y Recomendaciones}

Se obtuvieron dosificaciones mínimas y óptimas para la remediación de agua contaminada con colorantes. En dos diseños experimentales (exploración y optimización) se determinó que las dosis molares óptimas $\mathrm{Fe}^{+2} / \mathrm{H}_{2} \mathrm{O}_{2}$ para la decoloración de agua contaminada con amarillo 160, azul 81 y rojo 190 fueron 1:3 y 1:5 en medio ácido (pH 3,5). El consumo de peróxido de hidrógeno 30\% fue apenas de 3,04 $\mathrm{mL}$ por cada $250 \mathrm{~mL}$ de agua contaminada; es decir, se utilizó aprox. 1,2\% de peróxido de hidrógeno de la masa total de agua contaminada (DQO de $1719 \mathrm{mg} \mathrm{O}_{2} \cdot \mathrm{L}^{-1}$ ); mientras que el consumo de sulfato ferroso fue de apenas un $0,3 \%$ de la masa total del medio de reacción. 
Todas las variables respuesta (turbidez final, potencial de óxido-reducción, color y DQO) fueron medidas después de 24 horas.

Se experimentaron dos tipos de oxidantes (hipoclorito de sodio comercial al $5 \%$ y peróxido de hidrógeno al 30\%) para comparar su eficiencia con el reactivo Fenton. De estos tratamientos, el reactivo Fenton permitió la remoción del 99,9\% de DQO y 100\% de turbidez, en comparación con el cloro y el peróxido de hidrógeno los cuales no removieron ni el 50\% de la contaminación. De estos resultados se evidenció el poder oxidativo del reactivo Fenton para una contaminación de $3300 \mathrm{mg} . \mathrm{L}^{-1}$ de colorantes reactivos azoicos. El color final del agua tratada con dosis molares 1:3 y 1:5 no difiere significativamente del color del agua tipo I según análisis potenciométrico, colorimétrico y espectrofotométrico.

Además de procesos de coagulación química y natural, los procesos de oxidación avanzada ofrecen a la industria textilera una opción para el tratamiento de sus efluentes contaminados con residuos de colorantes reactivos azoicos. Efluentes de la industria textilera son considerados tóxicos y de alta carga contaminante. El proceso Fenton ha demostrado ser una opción económica en términos de consumo de reactivos y con amplias perspectivas de aplicación a gran escala en beneficio del medio ambiente.

\section{Bibliografía}

Arroyave Rojas, J. A., Garcés Giraldo, L. F., \& Mejía Trujillo, J. (2009). Emprego do reativo de Fenton para a degradação do corante Tartrazina. Revista Lasallista de Investigación, 6(1), 27-34.

Bahmani, P., Kalantary, R. R., Esrafili, A., Gholami, M., \& Jafari, A. J. (2013). Evaluation of Fenton oxidation process coupled with biological treatment for the removal of reactive black 5 from aqueous solution. Journal of Environmental Health Science and Engineering, 11(1), 13.

Barbusiński, K. (2005). The modified Fenton process for decolorization of dye wastewater. Polish Journal of Environmental Studies, 14(3), 281-285.

Benatti, C. T., \& Tavares, C. R. G. (2012). Fentons Process for the Treatment of Mixed Waste Chemicals. Faculdade Ingá-UNINGÁ, Universidade Estadual de Maringá-UEM, Brazil Unpublished, 25.

Bouasla, C., Ismail, F., \& Samar, M. E.-H. (2012). Effects of operator parameters, anions and cations on the degradation of AY99 in an aqueous solution using Fenton's reagent. Optimization and kinetics study. International Journal of Industrial Chemistry, 3(1), 1-11.

Chemizmu, K., \& Fentona, R. (2009). Fenton reaction-controversy concerning the chemistry. Ecological Chemistry and Engineering, 16, 347-358. 
Cui, D., Li, G., Zhao, M., \& Han, S. (2014). Decolourization of azo dyes by a newly isolated Klebsiella sp. strain Y3, and effects of various factors on biodegradation. Biotechnology \& Biotechnological Equipment, 28(3), 478-486. http://doi.org/10.1080/13102818.2014.926053

Daud, N. K., Akpan, U. G., \& Hameed, B. H. (2012). Decolorization of Sunzol Black DN conc. in aqueous solution by Fenton oxidation process: effect of system parameters and kinetic study. Desalination and Water Treatment, 37(1-3), 1-7. http://doi.org/ 10.1080/19443994.2012.661246

Domínguez, J. R., Muñoz, M. J., Palo, P., González, T., Peres, J. A., \& Cuerda-Correa, E. M. (2014). Fenton advanced oxidation of emerging pollutants: parabens. International Journal of Energy and Environmental Engineering, 5(2-3), 1-10. http://doi.org/10.1007/s40095-0140089-1

El Haddad, M., Regti, A., Laamari, M. R., Mamouni, R., \& Saffaj, N. (2014). Use of Fenton reagent as advanced oxidative process for removing textile dyes from aqueous solutions. $J$ Mater Environ Sci, 5, 667-674.

El Telégrafo. (2009). Industria del jean afecta al río de Patate. El Telégrafo. Guayaquil, Ecuador. Retrieved from http://goo.gl/eOXLBR

El Telégrafo. (2013). Taiwán ofrece asesoría para textileros de Pelileo. El Telégrafo. Guayaquil, Ecuador. Retrieved from http://www.telegrafo.com.ec/regionales/regionalcentro/item/taiwan-ofrece-asesoria-para-textileros-de-pelileo.html

Eslami, A., Moradi, M., Ghanbari, F., \& Mehdipour, F. (2013). Decolorization and COD removal from real textile wastewater by chemical and electrochemical Fenton processes: a comparative study. Journal of Environmental Health Science and Engineering, 11(1), 31.

Gaber, M., Abu Ghalwa, N., Khedr, A. M., \& Salem, M. F. (2013). Electrochemical Degradation of Reactive Yellow 160 Dye in Real Wastewater Using $\mathrm{C} / \mathrm{PbO}^{2-}, \mathrm{Pb}+\mathrm{Sn} / \mathrm{PbO}_{2}+\mathrm{SnO}^{2-}$, and $\mathrm{Pb} / \mathrm{PbO}_{2}$ Modified Electrodes. Journal of Chemistry, 2013, 1-9. http://doi.org/10.1155/2013/691763

Garcés Giraldo, L. F., \& Hernández Ángel, M. L. (2012). Tratamiento de aguas residuales de la industria textil por medio de luz ultravioleta: una comparación de dióxido de titanio y fenton. Retrieved from http://repository.lasallista.edu.co/dspace/handle/10567/701

Grekova-Vasileva, M., \& Topalova, Y. (2009). Biological Algorithms for Textile Wastewater Management. Biotechnology \& Biotechnological Equipment, 23(sup1), 442-447. http://doi.org/10.1080/13102818.2009.10818459 
Hansson, H., Kaczala, F., Marques, M., \& Hogland, W. (2012). Photo-Fenton and Fenton Oxidation of Recalcitrant Industrial Wastewater Using Nanoscale Zero-Valent Iron. International Journal of Photoenergy, 2012, 1-11. http://doi.org/10.1155/2012/531076

Kalra, S. S., Mohan, S., Sinha, A., \& Singh, G. (2011). Advanced Oxidation Processes for Treatment of Textile and Dye Wastewater: A Review. In 2nd International Conference on Environmental Science and Development (Vol. 4, pp. 271-5). Retrieved from http://www.ipcbee.com/vol4/59-ICESD2011D30020.pdf

Körbahti, B. K., \& Tanyolaç, A. (2008). Electrochemical treatment of simulated textile wastewater with industrial components and Levafix Blue CA reactive dye: Optimization through response surface methodology. Journal of Hazardous Materials, 151(2-3), 422-431. http://doi.org/10.1016/j.jhazmat.2007.06.010

Lau, Y.-Y., Wong, Y.-S., Teng, T.-T., Morad, N., Rafatullah, M., \& Ong, S.-A. (2014). Coagulationflocculation of azo dye Acid Orange 7 with green refined laterite soil. Chemical Engineering Journal, 246, 383-390. http://doi.org/10.1016/j.cej.2014.02.100

Litter, M. I., Domènech, X., \& Jardim, W. F. (2001). Procesos avanzados de oxidación para la eliminación de contaminantes. Eliminación de Contaminantes Por Fotocatálisis Heterogénea, 1. Retrieved from http://goo.gl/HHZtrn

Moreno, A., Figueroa, D., \& Hormaza, A. (2013). Adsorción de azul de metileno sobre cascarilla de arroz. Retrieved from http://repository.lasallista.edu.co/dspace/handle/10567/837

Ohura, S., Harada, H., Shiki, M., Kawakita, H., \& Biswas, B. K. (2012). Decoloration of Acid Red 88 Using Synthetic-Zeolite-Based Iron as a Heterogeneous Photo-Fenton Catalyst. Environment and Pollution, 2(1). http://doi.org/10.5539/ep.v2n1p36

Patel, R. D., \& Patel, R. L. (2013). Treatment of Dye Intermediate Waste-Water by Fenton and Electro-Fenton Treatments. International Journal of Research in Modern Engineering and Emerging Technology, 1(3), 7.

Revelo, A., Proaño, D., \& Banchón, C. (2015). Biocoagulación de aguas residuales de industria textilera mediante extractos de Caesalpinia spinosa. Enfoque UTE, $6(1), 1$ - 12.

Riaza Frutos, A., Manzano, M., \& Quiroga, J. (2007). Oxidación de azo-colorantes vía reacción foto-Fenton. Ingeniería Química. Facultad de Ciencias del Mar y Ambientales, Universidad de Cádiz. Retrieved from http://www.inese.es/html/files/pdf/amb/iq/444/IQfeb12.pdf

Rodriguez, R., Flesler, F., \& Lehmann, V. (2010). Oxidación avanzada - sistema Fenton - en tratamiento de efluentes industriales de alta carga orgánica. Inti Ambiente. 
Roy Choudhury, A. K. (2013). Green chemistry and the textile industry. Textile Progress, 45(1), 3143. http://doi.org/10.1080/00405167.2013.807601

Saber, A., Hasheminejad, H., Taebi, A., \& Ghaffari, G. (2014). Optimization of Fenton-based treatment of petroleum refinery wastewater with scrap iron using response surface methodology. Applied Water Science, 4(3), 283-290. http://doi.org/10.1007/s13201-0130144-8

Villacreces, L. (2013). Aplicación de oxidación química tipo fenton asistida con detergente para tratamiento de suelos contaminados con petróleo. Escuela Superior Politécnica del Ejercito. Retrieved from http://repositorio.espe.edu.ec/bitstream/21000/7802/1/T-ESPE047460.pdf 